\title{
Food Allergy: A Review
}

\author{
Mahboubeh Mansouri ${ }^{1 *}$ \\ ${ }^{1}$ Immunology and Allergy Department, Mofid Children's Hospital, Shahid Beheshti University of Medical Sciences, Tehran, IR Iran \\ ${ }^{*}$ Corresponding author: Mahboubeh Mansouri, Immunology and Allergy Department, Mofid Children's Hospital, Shahid Beheshti University of Medical Sciences, Tehran, IR Iran. \\ Fax:+98-2122227033, E-mail: mbmans65@gmail.com
}

Received: August 2, 2014; Revised: November 8, 2014; Accepted: December 11, 2014

\begin{abstract}
Context: Food allergy is a growing health problem. Avoidance of the food allergen is the only accepted treatment. Because the major food allergens are among the most commonly used foods including cow's milk, egg, nuts, wheat, soya, fish, and seafood, avoiding them is difficult and might negatively affect the patients' and their families' health.

Evidence Acquisition: This brief review concerns the prevalence, importance, definition, types, clinical symptoms, diagnosis, and management of food allergy. The information were retrieved searching a wide range of published data, especially in PubMed, from January 2000 through July 2014.

Results: Food allergies are mainly classified into IgE mediated and non-IgE mediated; the latter is classified into cell-mediated, and mixed IgE-non-IgE-mediated food allergy. Medical history can provide detailed information essential to make the diagnosis. The current approach to the management of food allergy substantially relies on allergen avoidance and prescriptions to treat allergic reactions.

Conclusions: The characteristic features of IgE-mediated food allergy are abrupt onset of clinical symptoms, which may result in a lifethreatening events, and positive results of the majority of the paraclinical tests that mainly trace the specific IgE to foods. Moreover, nonIgE-mediated food allergies present as chronic diseases and due to lack of proper diagnostic tests the similarity of the clinical presentation with other chronic clinical conditions, the exact prevalence may remain underestimated.
\end{abstract}

Keywords: Food Hypersensitivity; Hypersensitivity; Food

\section{Context}

Food allergy (FA) is a growing health problem with many important aspects. In one hand, it may have serious and life-threatening course that may lead to anaphylaxis, which imposes many health and psychologic problems to the patients and their families. On the other hand, it may present as a chronic illness, especially gastrointestinal (GI) problems, which may interfere with the child growth and development or mimic acute surgical conditions, other chronic GI diseases, or even metabolic and endocrine diseases. The main food allergens are the most commonly used nutrients in family's food baskets including cow's milk, egg, tree nuts, peanuts, wheat, soya, fish, and seafood. All these nutrients have their unique roles in the growth and development of children and facing FA can be a major challenge in the diagnosis and the management.

\section{Evidence Acquisition}

A broad scope of the literatures search, especially PubMed and American and European databases were the basis for this review. All recent published data including review articles written by the authorities in FA, the recently released guidelines, clinical trials, the epidemiologic studies in FA, the United States Food and Drug Administration's guidance in food allergens, mostly se- lected from January 2000 through July 2014, were investigated. This review was predominantly retrieved from two essential and recent guidelines in FA $(1,2)$

\section{Results}

The FA is an unfavorable immunologic reaction that cans repeatedly occur in response to the culprit food. The FA is a growing general health problem, which involves children and adults. It should be differentiate from nonimmunologic reactions, namely, food intolerance, which are mainly due to enzyme deficiency, metabolic disease, pharmacologic agent in food, and undefined mechanisms (3).

\subsection{Prevalence}

Recently, the prevalence of FA has been estimated at up to $10 \%$ of the population (4) and at around $8 \%$ in children. It is likely increased in the past decades (5). Food allergens are typically protein component of food that stimulate immune system and cause clinical reactions. Food allergens are mainly divided into class I, which can induce allergic reaction in raw and baked form foods, and class II food allergens, generally fruit and vegetables, that may result in hypersensitivity reactions when eaten raw (1). 


\subsection{Major Food Allergens}

All food may cause FA but Food and Drug Administration considers milk, fish, egg, shellfish, tree nuts, peanuts, soy, and wheat as the most important food allergens that account for $90 \%$ of reactions in young children (6). Of these, $2.2 \%, 1.8 \%, 1.7 \%$ are due to cow's milk, peanut, and tree nuts, respectively, are the most frequent allergens in children, and shellfish (1.9\%), fruits (1.6\%), and vegetables (1.3\%) are the most prevalent food allergens in adults (1). Steinke et al. reported milk as the most concerned allergen in children and as the second most prevalent food allergen in adults (7). Macdougall et al. cited milk as the cause of $10.9 \%$ of mortal anaphylactic reactions in the United Kingdom (8).

\subsection{Risk Factors}

The risk factors were as follows: sex (boys > girls and women > men); ethnicity (more prevalent in Asian and Africans); genetics; atopy; dietary fat content (intake of omega-3, long-chain fatty acids, and aryl hydrocarbon receptor (AHR) ligands suppress allergy; in contrast, high-fat diet and medium-chain triglycerides promote allergy); vitamins A and D insufficiency; antacid overconsumptions (causes allergen pass through the gut without digestion); diminished intake of antioxidants; obesity; and exposure to food allergens through non-oral routes (e.g. skin, particularly in the context of genetic barrier defects or inflamed skin) $(4,9)$.

Moreover, improved sanitation and the timing and route of exposure to food at first time (delaying introduction of food possibly increases environmental sensitization), genetic predispositions, infections, variations in the microflora of gut, maternal diet, antigen spread during lactation with breast milk, person's temperament, and the rate of antigen exposure are named as the important risk factors predicting food tolerance or sensitization (10).

The consistency of intestinal wall plays a critical role in directing the amount of allergens toward mucosal immune system, where the specialized regulatory $\mathrm{T}$ cells decide between oral tolerance development or hypersensitivity reaction, as well as beginning of both the IgE and non-IgE-mediated reactions $(11,12)$.

\subsection{Classifications}

The FA can be classified into three clusters of immunologic reactions including: IgE-mediated, non-IgE-mediated, and mixed type reactions (3).

\subsubsection{IgE-Mediated Food Allergy}

A defect in development or in a formerly well-established oral tolerance causes IgE-mediated FA. The synthetized food-specific IgE binds to mast cell basophil receptors and becomes in contact with the same offending food allergen again. The cross linkage of two specific IgE on these cells leads to release of histamine-like mediators, prostaglandins, leukotrienes, and cytokines. These events cause the immediate manifestations of FA. In the immediate symptoms of FA, furthermore, a late-phase reaction occurs in two to eight hours of the acute phase (13).

The FA is considered IgE-mediated reaction if the clinical symptoms begin shortly (within minutes up to two hours) after eating a given food with involvement of one or more organ systems such as skin (e.g. urticaria and angioedema), respiratory system (e.g. rhinitis and bronchospasm), GI tract, which may encompass the whole system (e.g. nausea, vomiting, diarrhea, and abdominal cramps), and cardiovascular system (e.g. cardiac arrhythmia or hypotension). This type of FA can be life-threatening and lead to anaphylaxis; therefore, collapse, syncope or incontinence are the hallmarks of the most severe forms (14).

In addition to these symptoms, IgE-mediated FA can present as following conditions:

i) Immediate GI hypersensitivity in which upper GI symptoms may occur within minutes and lower GI symptoms may occur either immediately or with a delay of up to several hours.

ii) Food-dependent exercise-induced anaphylaxis are occurrence of allergic reactions in simultaneous consumption of allergen food and doing exercise, usually within two hours. If either of these conditions is absent, the reaction will not happen.

iii) Oral allergy syndrome (OAS) is restricted symptoms such as itching in lips, mouth, and throat after taking raw fruits or vegetables with a structural homology to pollens in patients who are allergic to pollen.

iv) IgE-mediated cutaneous reactions to foods such as isolated urticaria, flushing, and angioedema, although is not very common, can also develop. The various-sized wheals establish rapidly after intake of the culprit food. Chronic urticaria rarely has food origin. Typically, IgEmediated food-induced anaphylaxis is believed to involve systemic mediator release from sensitized mast cells and basophils (1).

\subsubsection{Non-IgE-Mediated and Cell-Mediated Food Allergy: Gaterointestinal Symptoms}

These conditions have delayed onset or present as a chronic disease and are mostly confined to GI tract. The allergic symptoms develop two hours following eating the culprit food. The results of allergic test such as skin prick test and serum specific IgE to food are often negative; hence, they are considered cell-mediated allergy (15). These belated GI manifestations may indicate four groups of clinical symptoms:

i) Food protein-induced enterocolitis (FPIES): Clinical reactions present with projectile and persistent vomiting, Copious diarrhea consisted of blood, abdominal distention, lethargy, and weight loss, which may cause severe dehydration and shock in $20 \%$ of cases and maybe misdiagnosed as septicemia. The FPIES appears in the first month of life. The infant looks ill. The disease is mainly provoked by cow's milk and soy but cereals and poultry are also implicated. 
In these situations, breast milk and extensively hydrolyzed casein formula are recommended. It seems that an imbalance between the responses of tumor necrosis factor alpha (TNF- $\alpha$ ) and transforming growth factor beta (TGF- $\beta$ ) to food allergen plays a role in the pathogenesis. Although clinical manifestation is very sever in FPIES, physicians unawareness of the disease often causes extensive and unnecessary evaluation leading to the delayed diagnosis.

ii) Food protein-induced proctocolitis syndrome: Typically appears in the first two to four months of life in a healthyappearing breastfed infant. Stool contains streaks of blood. Biopsies of the rectum and sigmoid indicate eosinophilic infiltration in the tissue. Cow's milk and soy protein have the etiologic role in the disease. The bleeding is ceased three to four days after avoidance of the offending food.

iii) Food protein-induced enteropathy syndrome is intestinal inflammation triggered by cow's milk that presents with protracted diarrhea and sometimes vomiting and results in malabsorption syndrome and failure to thrive.

iv) Celiac disease: (gluten-sensitive enteropathy) is an extensive enteropathy leading to malabsorption. Total villous atrophy and an extensive cellular infiltrate are associated with sensitivity to gliadin. Patients present with diarrhea or sever steatorrhea, abdominal distention and flatulence, failure to thrive, and sometimes nausea and vomiting. Oral ulcers and other extra-intestinal symptoms secondary to malabsorption also occur (16).

\subsubsection{Non-IgE-Mediated and Cell-Mediated Food Allergy: Non Gaterointestinal Symptoms}

ia) Heiner's syndrome has been described as a rare disease in infant and young children, which depends on cow's milk ingestion, causing occult GI blood loss, anemia, pulmonary hemosiderosis, and failure to thrive. The condition is associated with the evidence of nonIgE-mediated immune responses, such as precipitating antibodies to milk protein. Combined cellular and immune-complex reactions are supposed to play a role in the pathogenesis of the disease (1).

ib) Asthma: With exception of accompanying of respiratory symptoms, asthma might present with an IgEmediated food reaction, which may take place along with other allergic manifestation (skin, cardiovascular, and GI tract involvements). The correlation between FA and asthma is a matter of debate. It is believed that asthma and FA have some associations. First, poorly controlled asthma is a risk factor for fatal food-induced anaphylactic reactions (17). Second, FA may induce airway hyper-reactivity in sensitized subjects (18). Finally, food allergy is considered as a major risk factor for severe lifethreatening asthma in children (19).

\subsubsection{Mixed IgE- and Non-IgE-Mediated Food Allergy}

This concept has also a delayed or chronic onset. It might present as atopic dermatitis (AD) and as eosinophilic gastroenteropathy (14). i) AD: Skin barrier dysfunction in a complex association with the environmental factors such as irritants, microbes, and allergens, plays a critical role in the pathogenesis of the illness. Null mutations of the skin barrier protein filaggrin triggers transcutaneous allergen sensitization and the evolution of food allergy (1). About 35\% of children with moderate to severe AD have FA(20). Egg, milk, and peanut constitute $75 \%$ of food allergens, which may lead to $\mathrm{AD}$ (13).

ii) Allergic eosinophilic esophagitis and gastroenteritis: the disease is defined by infiltration of GI tract with eosinophils. Depending on the location of eosinophils, symptoms may vary. Gastroesophageal reflux, emesis, dysphagia, abdominal pain, and food impaction indicate esophageal involvement. About one half of patients with allergic eosinophilic esophagitis (AEE) and allergic eosinophilic gastritis (AEG) have peripheral eosinophilia, and patients with severe AEG might have anemia, blood in the stool, and decreased serum protein, albumin, and IgG levels. The pathogenesis has not been defined; however, food and aeroallergens are implicated and elimination diets lead to clinical improvements. Failure to thrive, anemia, and protein-losing enteropathy are among other clinical manifestations related to GI tract involvement $(2,21)$.

\subsection{Diagnosis}

The guidelines of National Institute of Allergy and Infectious Diseases (NIAID) and clinical guideline 116 of National Institute for Health and Care Excellence (NICE) recommend that FA should be considered in any individual presenting with anaphylaxis or any immediate combination of symptoms that occur after ingestion of food (IgEmediated FA) (1). FA can also be considered when each of the symptoms contained in the right column of Table 1 develops in a chronic condition (Non-IgE-mediated FA).

Medical history can provide detailed information essential to make the diagnosis. A physician should get a thorough medical history including information regarding suspected food triggers, the quantity of foods needed to provoke reactions, the spectrum of observed symptoms, the interval between eating the contaminated food and the onset of symptoms, reproducibility of reactions, and accompanying cofactors such as alcohol use, exercise, or drug consumption (22).

i) The laboratory tests that trace the specific IgE to food include skin prick test, radioallergosorbent test (RAST), enzyme-linked immuno assay (ELISA), food-specific IgE antibodies (e.g. catabolite activator protein (CAP) system, fluorescent enzyme immunoassay (FEIA), a specific IgE (sIgE) microarray (Phadia Isac) allowing the detection of sIgE for a whole batch of food and aeroallergens (23), or an indirect approach to sensitization by a basophil activation test using flow cytometry, which assesses the percentage of basophils bearing sIgE activation and expresses CD63 marker after in vitro stimulation with allergens $(24,25)$.All the test are developed along with medical history to validate or invalidate an IgE mediated FA. 


\begin{tabular}{|c|c|c|}
\hline Alergy Organ & IgE-Mediated & Non-IgE-mediated \\
\hline \multicolumn{3}{|l|}{ The skin } \\
\hline & Pruritus & Pruritus \\
\hline & Erythema & Erythema \\
\hline & Acute urticarial (localized or generalized) & Atopic eczema \\
\hline & $\begin{array}{c}\text { Acute angioedema, most commonly of lips, face, and } \\
\text { around eyes }\end{array}$ & \\
\hline \multicolumn{3}{|c|}{ The gastrointestinal system } \\
\hline & Angioedema of the lips, tongue, and palate & Oral pruritus \\
\hline & Oral pruritus & Loose or frequent stools \\
\hline & Nausea & Blood and/or mucus in stool \\
\hline & Colicky abdominal pain & Colicky abdominal pain \\
\hline & Vomiting & Infantile colic \\
\hline & Diarrhea & Food refusal or aversion \\
\hline & & Constipation \\
\hline & & Perianal redness \\
\hline & & Pallor and tiredness \\
\hline & & $\begin{array}{l}\text { Growth faltering in conjunction } \\
\text { with at least one or more of the } \\
\text { above gastrointestinal symptoms } \\
\text { (with or without significant } \\
\text { atopic eczema) }\end{array}$ \\
\hline
\end{tabular}

The respiratory system ${ }^{a}$

Upper respiratory tract symptoms (nasal itching, sneezing, rhinorrhea, or congestion [with or without conjunctivitis])

Lower respiratory tract symptoms (cough, chest tightness, wheezing, or shortness of breath)

Others

Signs or symptoms of anaphylaxis or other systemic allergic reactions

\footnotetext{
${ }^{\mathrm{a}}$ Usually in combination with one or more of the above symptoms and signs.
}

ii) Skin prick tests (SPTs), when the results are positive, provide a rapid mean to detect sensitization for IgE-mediated disorders. A positive result for SPT, with specificity of $100 \%$, does not necessarily verify that the food is the causative agent. In contrast, a negative result of SPT, with a negative predictive value of $90 \%$, suggests the absence of IgE-mediated allergic reactivity. Consequently, with consideration of medical history, a clinician might conclude that an allergen-sIgE level, which has $60 \%$ positive predictive value, is sufficient to make the diagnosis of clinical FA. Allergens eliciting a wheal at least $3 \mathrm{~mm}$ larger than the one produced by the negative control are considered to have positive results, indicating the possibility that the patient has symptomatic reactivity to the specific food, with strongly positive results. Recent studies suggest the contribution of a free light-chain immunoglobulin fluorescent enzyme immunoassay in addition to IgE in the allergic reaction to cow's milk proteins (CMP) (26). In addition, a number of studies have refined the method of using available tests including the next generation of sIgE tests, which evaluate "components" or specific proteins within foods, often termed component-resolved diagnostics (CRD) (4).

iii) Atopy patch test is performed by placing foods under fin chambers for 48 hours, then removing the chamber and reading the skin modifications 24 hours later. The atopy patch test seems promising, particularly in identifying foods that might be eliciting non-IgE-mediated reactions; however, there are currently no standardized reagent, method of application, or interpretation (27).

Endoscopy and biopsy are the most definitive approaches for diagnosing many GI hyper sensitivities. Greater than 15 to 20 eosinophils per $40 \times$ high-power field in the esophagus is diagnostic of AEE, especially if the $\mathrm{pH}$ probe is within normal limits and there is a lack of response to anti-reflux medication. Eosinophils are normally present in the gastric and intestinal mucosa; therefore, eosinophil numbers must be greater to make the diagnosis of 
AEG. In patients with suspected celiac disease, the presence of serum IgA anti-tissue transglutaminase (antiendomysium) and antigliadin antibodies of greater than $90 \%$ are predictive of celiac disease (2).

iv) Oral food challenge (OFC): The double-blind placebo-controlled food challenge (DBPCFC) is the gold standard in diagnosis of FA; however, a single-blind or an open-food challenge may be considered diagnostic if the objective symptoms correlate with medical history and are supported by laboratory tests results. During the OFC, the offending food is given under strict medical supervision in order to determine tolerance or clinical reactivity. There is a risk of severe reaction; thus, the medical staff must be properly trained with medications and equipment to treat anaphylaxis on hand, according to validated and well-known procedures (28). Since, food challenges in an IgE mediated setting can elicit severe, and even life-threatening reactions they should be performed by qualified personnel (29). Patients with history of life-threatening anaphylaxis should be challenged only when the history and laboratory testing cannot conclusively determine the causative food or the patient is believed to have developed clinical tolerance (2). To avoid performing challenge test as a troublesome and timeconsuming procedure, quantitative measurement of food-specific IgE antibodies (e.g. CAP System FEIA, Pharmacia-Upjohn Diagnostics) is more predictive of symptomatic IgE-mediated FA. The cutoff points of the specific IgE for egg, cow's milk, peanut, fish, and tree nuts have been established and are related to clinical symptoms with predictive value greater $>95 \%$ (2).

\subsection{Diagnosis of Non-IgE-Mediated Food Allergy}

Generally, non-IgE allergies are underrepresented in the FA dialogue (30). Based on the results of the allergyfocused clinical history, if non-IgE-mediated FA is suspected, trial elimination of the suspected allergen (normally between two and, six weeks) and reintroduction after the trial will follow. The therapist must seek advice from a dietitian with appropriate competency about nutritional adequacies, timings of elimination and reintroduction, and follow-up (21). The afore mentioned screening tests, e.g. SPTs, sIgE tests, and atopy patch tests (APTs), lack specificity and sensitivity (31).

Diet diaries can be a useful supplement to medical history, especially in chronic disorders. Many young children with AEE and all of patients with AEG have multiple food allergies and consequently, it is necessary to administer an elemental formula (i.e. Neocate or Ele Care) for four to six weeks before initiating the challenges. An open or singleblinded food challenge are often used to screen potential allergens in AEE and AE.G. especially because it might take repeated feedings over few days to elicit symptoms (2).

\subsection{Natural Course of Food Allergy}

Typically, childhood food allergies to milk, egg, wheat, and soy resolve during childhood, whereas allergies to peanut, tree nuts, fish, and shellfish are persistent (4). Prognosis also varies by disorder; e.g. Food-related AEE seems to have relatively poor chance of resolution (32). However, there is evidence of further delay in occurring natural tolerance to foods comparing the previous reports (4). Factors that are significantly associated with tolerance development include mild to moderate reaction history, early lower sIgE levels, having only one FA, presenting with only $\mathrm{AD}$, having a white versus black ethnicity, and presenting at younger age with the first reaction. It is beneficial for a patient that has food allergy and who met the above conditions, to re-enter the culprit food (after a period of avoidance of course) every other time, rather than the avoidance for a long period of time without re-trying the food (33).

\subsection{Management/Treatment}

The current approach to manage FA substantially relies on allergen avoidance, preparations to promptly treat allergic reactions, prescription of self-injectable epinephrine, and education about prehospital treatment of reactions and allergen avoidance (1). If a food-elimination diet is advised as part of the diagnostic process, the physician should consider the socioeconomic status, cultural, and religious issues of the child or young person and their parent or caregiver in offering information on what foods and drinks to avoid, how to interpret food labels, alternative sources of nutrition to ensure adequate nutritional intake, the safety and limitations of an elimination diet, the proposed duration of the elimination diet, and when, where, and how an oral food challenge or food reintroduction procedure may be undertaken (21).

There has been an increased emphasis on developing immunotherapeutic approaches to treat FA in past decade. Traditional standard subcutaneous immunotherapy used to treat pollen and insect allergies was found to provoke severe adverse reactions with food (34). Oral immunotherapy (OIT) has been most intensively investigated over the past decade and in small, mostly uncontrolled, trials has been shown to induce "desensitization". Although OIT shows promise as an effective form of therapy, the high rate of adverse reactions and doubt of long-term outcome mandate further study (35).

According to evidences, a majority of children with allergy to egg or cow's milk protein allergy (CMA) can extensively tolerate heated forms of these allergens such as bakery forms, and ingestion of these products might speed recovery. Although not initially considered an immunotherapeutic approach, it is a major progress in management of allergic disorders (2).

\section{Conclusions}

Reviewing all subtopics in FA, from definition to managements, the lack of studies in this field in Iran became more obvious. The mechanism of FA is categorized into 
IgE-mediated and non-IgE-mediated; these mechanisms are different in the interval between the entrance of the culprit food and the beginning of clinical presentation as well as the type of clinical reactions. The elapsing time is within two hours after taking the meal in IgE-mediated reactions while in non-IgE-mediated reactions, it may take four hours or even several days after ingestion of the allergenic food to happen. The clinical symptoms are also different in these two kinds of allergic reactions to food. In the IgE-mediated reactions, the clinical presentations have abrupt onset and can be life threatening, whereas in the non-IgE-mediated reactions, the clinical symptoms are often limited to the GI tract or even mimic other clinical conditions such as metabolic disorders and surgical conditions; moreover, they may cause malnutrition and failure to thrive. Due to the lack of the proper diagnostic tests regarding the non-IgE-mediated FA and prolonged interval between food entrance and the clinical presentations, the diagnosis seems difficult and mostly relies on physician index of suspicion and trying the avoidance of suspected foods for at least six to eight weeks and following the consequent clinical responses.

As a result, one can draw a conclusion that since nonIgE-mediated FA is more difficult to diagnose than IgEmediated one, the prevalence of the non-IgE-mediated FA might be underestimated. Finally, another important aspect of FA is that the major food allergen are the food that are most commonly used including cow's milk, egg, nuts, soya, wheat, and sea products, which makes them very hard to avoid; therefore, the management of the condition is challenging.

\section{Acknowledgements}

I appreciate Pediatric Infectious Disease Research Center of Shahid Beheshti University of Medical Sciences for its general support.

\section{Authors' Contributions}

Dr Mahboubeh Mansouri is the only one responsible for gathering the data, reviewing the articles, and preparing the manuscript.

\section{Financial Disclosure}

The author declared no financial interests related to the material in the manuscript.

\section{Funding/Support}

This study was not supported neither financially nor materially by any organization.

\section{References}

1. Boyce JA, Assa'ad A, Burks AW, Jones SM, Sampson HA, Wood RA, et al. Guidelines for the diagnosis and management of food allergy in the United States: summary of the NIAID-Sponsored Expert Panel report. JAm Acad Dermatol. 2011;64(1):175-92.
2. Sampson HA. Update on food allergy. J Allergy Clin Immunol. 2004;113(5):805-19.

3. Clarke S. Diagnosis and assessment of food allergy in children and young people. Community Pract. 2011;84(6):39-41.

4. Sicherer SH, Sampson HA. Food allergy: Epidemiology, pathogenesis, diagnosis, and treatment. J Allergy Clin Immunol. 2014;133(2):291-307.

5. Shaker M. New insights into the allergic march. Curr Opin Pediatr 2014;26(4):516-20.

6. U.S. Food and Drug Administration.. Food allergens guidance documents and regulatory information. 2013. Available from: http:/| www.fda.gov/Food/GuidanceRegulation/GuidanceDocumentsRegulatoryInformation/Allergens/default.htm.

7. Steinke M, Fiocchi A, Kirchlechner V, Ballmer-Weber B, Brockow $\mathrm{K}$, Hischenhuber $\mathrm{C}$, et al. Perceived food allergy in children in 10 European nations. A randomised telephone survey. Int Arch Allergy Immunol. 2007;143(4):290-5.

8. Macdougall CF, Cant AJ, Colver AF. How dangerous is food allergy in childhood? The incidence of severe and fatal allergic reactions across the UK and Ireland. Arch Dis Child. 2002;86(4):236-9.

9. Visness CM, London SJ, Daniels JL, Kaufman JS, Yeatts KB, SiegaRiz AM, et al. Association of obesity with IgE levels and allergy symptoms in children and adolescents: results from the National Health and Nutrition Examination Survey 2005-2006.J Allergy Clin Immunol. 2009;123(5):1163-9.

10. Karlsson MR, Rugtveit J, Brandtzaeg P. Allergen-responsive CD4+CD25+ regulatory T cells in children who have outgrown cow's milk allergy.J Exp Med. 2004;199(12):1679-88.

11. Nagata S, McKenzie C, Pender SL, Bajaj-Elliott M, Fairclough PD, Walker-Smith JA, et al. Human Peyer's patch T cells are sensitized to dietary antigen and display a Th cell type 1 cytokine profile. $J$ Immunol. 2000;165(9):5315-21.

12. Venter C, Groetch M. Nutritional management of food protein induced enterocolitis syndrome. Curr Opin Allergy Clin Immunol. 2014;14(3):255-62.

13. Story RE. Manifestations of food allergy in infants and children. Pediatr Ann. 2008;37(8):530-5.

14. Fiocchi A, Brozek J, Schunemann H, Bahna SL, von Berg A, Beyer $\mathrm{K}$, et al. World Allergy Organization (WAO) Diagnosis and Rationale for Action against Cow's Milk Allergy (DRACMA) Guidelines. World Allergy Organ J. 2010;3(4):57-161.

15. Norwak-Wegrzyn A. Food protein-induced enterocolitis, enteropathy, and proctocolitis. In: Metcalfe DD, Sampson HA, Simon RA editors. Food Allergy: adverse reactions to foods and food additives. 3rd ed. Baltimore: Blackwell Publishing Inc; 2003. pp. 227-41.

16. Perry TT, Pesek RD. Clinical manifestations of food allergy. Pediatr Ann. 2013;42(6):96-101.

17. Pumphrey RSH. Anaphylaxis. John Wiley \& Sons, Ltd; 2008. pp. 116-32.Fatal Anaphylaxis in the UK, 1992-2001.

18. James JM, Bernhisel-Broadbent J, Sampson HA. Respiratory reactions provoked by double-blind food challenges in children. Am J Respir Crit Care Med. 1994;149(1):59-64.

19. Roberts G, Patel N, Levi-Schaffer F, Habibi P, Lack G. Food allergy as a risk factor for life-threatening asthma in childhood: a casecontrolled study.J Allergy Clin Immunol. 2003;112(1):168-74.

20. Thompson MM, Tofte SJ, Simpson EL, Hanifin JM. Patterns of care and referral in children with atopic dermatitis and concern for food allergy. Dermatol Ther. 2006;19(2):91-6.

21. National Institute for Health and Care Excellence.. Food allergy in children and young people. 2011. Available from: www.nice.org. uk/cg116.

22. Skypala I. Adverse food reactions--an emerging issue for adults. Am Diet Assoc. 2011;111(12):1877-91.

23. Sanz ML, Blazquez AB, Garcia BE. Microarray of allergenic component-based diagnosis in food allergy. Curr Opin Allergy Clin Immunol. 2011;11(3):204-9.

24. Wanich N, Nowak-Wegrzyn A, Sampson HA, Shreffler WG. Allergen-specific basophil suppression associated with clinical tolerance in patients with milk allergy. J Allergy Clin Immunol. 2009;123(4):789-94 e20.

25. Rubio A, Vivinus-Nebot M, Bourrier T, Saggio B, Albertini M 


\section{Mansouri M}

Bernard A. Benefit of the basophil activation test in deciding when to reintroduce cow's milk in allergic children. Allergy. 2011;66(1):92-100.

26. Schouten B, van Esch BC, van Thuijl AO, Blokhuis BR, Groot Kormelink T, Hofman GA, et al. Contribution of IgE and immunoglobulin free light chain in the allergic reaction to cow's milk proteins. J Allergy Clin Immunol. 2010;125(6):1308-14.

27. Kalach N, Soulaines P, de Boissieu D, Dupont C. A pilot study of the usefulness and safety of a ready-to-use atopy patch test (Diallertest) versus a comparator (Finn Chamber) during cow's milk allergy in children.J Allergy Clin Immunol. 2005;116(6):1321-6.

28. Flinterman AE, Knulst AC, Meijer Y, Bruijnzeel-Koomen CA, Pasmans SG. Acute allergic reactions in children with AEDS after prolonged cow's milk elimination diets. Allergy. 2006;61(3):370-4.

29. Perry TT, Matsui EC, Conover-Walker MK, Wood RA. Risk of oral food challenges. J Allergy Clin Immunol. 2004;114(5):1164-8.

30. Schultz F, Westcott-Chavez A. Food protein-induced enterocolitis syndrome from the parent perspective. Curr Opin Allergy Clin Immunol. 2014;14(3):263-7.

31. Dominguez-Ortega G, Borrelli O, Meyer R, Dziubak R, De Koker $\mathrm{C}$, Godwin $\mathrm{H}$, et al. Extraintestinal manifestations in children with gastrointestinal food allergy. J Pediatr Gastroenterol Nutr. 2014;59(2):210-4.

32. DeBrosse CW, Franciosi JP, King EC, Butz BK, Greenberg AB, Collins $\mathrm{MH}$, et al. Long-term outcomes in pediatric-onset esophageal eosinophilia. J Allergy Clin Immunol. 2011;128(1):132-8.

33. Burks AW, Land MH. Long-term follow-up of IgE-mediated food allergy: determining persistence versus clinical tolerance. Ann Allergy Asthma Immunol. 2014;112(3):200-6.

34. Cox L, Nelson H, Lockey R, Calabria C, Chacko T, Finegold I, et al Allergen immunotherapy: a practice parameter third update. $J$ Allergy Clin Immunol. 2011;127(1 Suppl):S1-55.

35. Sampson HA. Peanut oral immunotherapy: is it ready for clinical practice? J Allergy Clin Immunol Pract. 2013;1(1):15-21. 